\title{
ASSOCIAÇÃO ENTRE OS SINTOMAS DA DISFUNÇÃO TEMPOROMANDIBULLAR E SUA RELAÇÃO COM FATORES PSICOLÓGICOS EM COMUNIDADES DE CUIABÁ-MT
}

\section{ASSOCIATION BETWEEN THE SYMPTOMS OF TEMPOROMANDIBULAR DYSFUNCTION AND ITS RELATIONSHIP WITH PSYCHOLOGICAL FACTORS IN CUIABÁ-MT COMMUNITIES}

\author{
Gilmar Jorge de Oliveira Júnior \\ José Nilton da Cruz* \\ Laiane Ditos"** \\ Layla Nayane dos Santos Candido**** \\ Lorena Frange Caldas
}

\begin{abstract}
RESUMO
Introdução: A articulação temporomandibular (ATM) estabelece a ligação móvel entre o osso temporal e a mandíbula, é uma articulação do tipo sinovial, que se inter-relaciona anatômica e cinesiologicamente com as articulações adjacentes e da coluna cervical. Entre as diversas alterações da ATM, encontram-se as desordens temporomandibulares (DTM), termo este empregado para unir um grupo de problemas clínicos de articulação, dos músculos mastigatórios e estruturas situadas em local próximo. A presença dos sintomas psicológicos pode gerar consequências para o indivíduo e o grupo social em que vive. Objetivo: O presente estudo tem por objetivo analisar a prevalência de DTM nos moradores das comunidades de Cuiabá-MT e sua associação com fatores psicológicos. Metodologia: Trata-se de um estudo exploratório transversal, de abordagem quanti-qualitativa, realizada no município de Cuiabá-MT, com 120 voluntários de ambos os sexos. Resultados: Mostraram que há uma maior prevalência em indivíduos do sexo feminino, com companheiro, que trabalham e estudam e não católicos. Discussão: No que se diz respeito ao questionário EADS-21, a depressão teve maior destaque, para o SF-36 a única variável que não apresentou associação foi à limitação por aspecto físico. Conclusão: Os resultados indicaram que a prevalência dessa doença pode ser alta, tendo relação com fatores psicológicos.

DESCRITORES: Índice Anamnésico de Helkimo • Fatores psicológicos • Qualidade de vida • Teste de Kruskal-Wallis.
\end{abstract}

\section{ABSTRACT}

Introduction: The temporomandibular joint (TMJ) establishes a mobile link between the temporal bone and jaw, it is a joint synovial type, which interrelates anatomically and cynesiologically with the adjacent joints and cervical spine. Among the various changes of the TMJ are temporomandibular disorders (TMD), which is a term used to join a group of clinical problems of articulation, masticatory muscles and structures situated next location. The presence of psychological symptoms may have consequences for the individual and the social group where he lives. Objective: This study aims to analyze the prevalence of TMD in residents of Cuiabá communities, and their association with psychological factors. Methodology: This is a cross-sectional exploratory study of quantitative and qualitative approach, held in the city of Cuiabá, with 120 volunteers of both sexes. Results: The results showed that there is a higher prevalence in females, with a partner, who works and studies and non-catholics. Discussion: As regards the EADS-21 questionnaire, depression was more prominent for the SF36; the only variable that showed no association was the limitation of physical appearance. Conclusion: The results indicated that the prevalence of this disease can be high, being related to psychological factors.

DESCRIPTORS: Anamnesic of Helkimo Index • Psychological factors • Quality of life • Kruskal-Wallis test.

* Universidade Federal de Mato Grosso, Professor, gilmarjr@ufmt.br.

** Universidade Federal de Mato Grosso, Professor, niltonn.cruz@gmail.com.

*** Universidade de Cuiabá, Fisioterapeuta, laianne.ditos@gmail.com.

**** Universidade de Cuiabá, Fisioterapeuta, laylla.nayane@gmail.com.

***** Universidade de Cuiabá, Professora, lorenafcaldas@hotmail.com. 


\section{N T RO DUÇÃO}

Sabe-se que a articulação temporomandibular (ATM) estabelece a ligação móvel entre o osso temporal e a mandíbula'. É uma articulação do tipo sinovial, que se relaciona anatômica e cinesiologicamente com as articulações adjacentes e da coluna cervical ${ }^{2}$. Essa articulação está entre uma das mais usadas no corpo humano, se movimenta de 1500 a 2000 vezes por dia, pois é responsável por funções importantes como morder, pronunciar, bocejar, engolir, inspirar e expirar ${ }^{3}$. Entre as diversas alterações da ATM, encontra-se a disfunção temporomandibular (DTM) ${ }^{4,5}$.

A DTM é um termo empregado para unir um grupo de problemas clínicos de articulação, dos músculos mastigatórios e estruturas situadas em local próximo ${ }^{6}$. Manifesta origem multicausal, não sendo possível reconhecer um único elemento etiológico causador da disfunção, sendo que esta é originada por associação entre fatores psicológicos que desequilibram os músculos mastigatórios e a articulação temporomandibular em $\mathrm{si}^{7,8}$.

A incidência desse tipo de disfunção vem aumentando significativamente, estima-se que mais de $60 \%$ da população já exibiu pelo menos um sinal de DTM em algum momento de sua vida, mas, por uma parcela mínima desses indivíduos necessitarem de tratamento, não se tem uma informação mais precisa da quantidade de pessoas que apresentam o distúrbio 9 .

Essas disfunções são caracterizadas por um conjunto de sinais e sintomas que podem conter ruídos articulares, dor nos músculos da mastigação, diminuição dos movimentos mandibulares, dores faciais, cefaleias e dores na articulação temporomandibular $^{10}$. Esse desarranjo na articulação é considerado a causa mais frequente de dor orofacial crônica ${ }^{11}$.

A presença dos sintomas psicológicos como estresse, ansiedade, depressão e baixa qualidade de vida podem intervir no início e na manutenção da dor crônica $^{12}$ e gerar consequências para o indivíduo, para sua família, seu trabalho e grupo so-

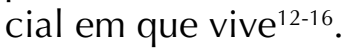

Diante disso, o presente estudo tem por objetivo analisar a prevalência de DTM na população da cidade de Cuiabá-MT, e sua associação com fatores psicológicos, como estresse, ansiedade, depressão e qualidade de vida.

\section{METODOLOGIA}

\section{Participantes e Tipo de Estudo}

Trata-se de um estudo exploratório transversal, de abordagem quanti-qualitativa, em que os indivíduos participantes voluntários eram de ambos os sexos, com idade entre 18 e 59 anos, moradores do município de Cuiabá-MT e que trabaIhavam e/ou estudavam. Foram excluídos indivíduos voluntários com idade menor que 18 anos porque a dentição pode não estar completa e voluntários com idade maior que 59 anos, pois essa pesquisa não englobou pacientes idosos, pessoas que apresentem sequelas neurológicas, uma vez que esses não têm autonomia para responder os questionários aplicados. Adicionalmente, as gestantes, em razão de poderem apresentar dores que sejam próprias da gestação e não com relação à disfunção, e pós-operatório de cirurgias craniomandibulares também não participaram deste estudo.

A amostragem realizada foi de conveniência e o tamanho da amostra atendeu às especificações de Dancey e Reidy ${ }^{17}$, que preveem um número mínimo de 100 participantes na análise e haver pelo menos cinco vezes mais participantes do que variáveis. Com base nos instrumentos recomendados, têm-se 21 variáveis (domínios), ou seja, a amostra de 120 indivíduos desta pesquisa ultrapassou os critérios especificados.

\section{Instrumento}

Foram utilizados os questionários: Anamnésico de Fonseca para avaliar e caracterizar a severidade dos sintomas da disfunção temporomandibular (DTM), EADS-21 que determina a escala de ansiedade, depressão e stress e o SF-36 para mensurar a qualidade de vida dos indivíduos. O questionário como um todo foi composto por quatro partes distintas: na primeira, foram coletados os dados sociodemográficos dos sujeitos e nas etapas seguintes decorreram as aplicações dos questionários citados anteriormente em sua respectiva sequência.
OLIVEIRA JÚNIOR GJ CRUZ JN

DITOS L

CANDIDO LNS

CALDAS LF

ASSOCIAÇÃO ENTRE

OS SINTOMAS

DA DISFUNÇÃO

TEMPOROMANDIBULAR

e sua relação

COM FATORES

PSICOLÓGICOS EM

COMUNIDADES DE

CUIABÁ-MT
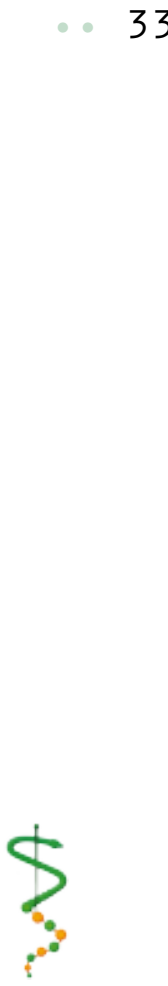

REV, ODONTOL.

UNIV. CID. SÃO

PAULO

$2016 ; 29(1)$ : 32 -

41 , JAN-ABR 
OLIVEIRA JÚNIOR GJ

CRUZ JN

DITOS L

CANDIDO LNS

CALDAS LF :

ASSOCIAÇÃO ENTRE

OS SINTOMAS

DA DISFUNÇÃO

TEMPOROMANDIBULAR

E SUA RELAÇÃO

COM FATORES

PSICOLÓGICOS EM

COMUNIDADES DE

CUIABÁ-MT

34

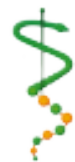

REV, ODONTOL.

UNIV, CID, SÃO

PAULO

$2016 ; 29(1): 32$ -

41, JAN-ABR
O questionário Anamnésico Fonseca consiste em uma versão modificada do Índice Anamnésico de Helkimo, e é um dos poucos instrumentos disponíveis em língua portuguesa que avalia e caracteriza a severidade dos sintomas da disfunção temporomandibular (DTM) ${ }^{18,19}$. Esse instrumento é composto por 10 perguntas e, para cada uma das questões, são possíveis três respostas: NÃO, ÁS VEZES e SIM, para as quais são preestabelecidas pontuações: 10 , 0 e 5, respectivamente. Com a somatória dos pontos atribuídos, obtém-se um índice anamnésico que permite classificar os voluntários em categorias de severidade de sintomas: sem DTM (0 a 15 pontos), DTM leve (20 a 45 pontos), DTM moderada (50 a 65) e DTM severa (70 a 100 pontos).

O outro instrumento utilizado, o questionário EADS-21, organiza-se em três escalas: Depressão, Ansiedade e Stress, contemplando sete itens para cada uma delas, totalizando 21 itens. Cada item consiste em uma frase, uma afirmação que remete para sintomas emocionais negativos. Para cada frase existem quatro possibilidades de resposta, "não se aplicou nada a mim", "aplicou-se a mim algumas vezes", "aplicou-se a mim muitas vezes", "aplicou-se a mim a maior parte das vezes", transformadas em notas de 0 a 3, respectivamente. Os resultados de cada escala são determinados pela soma dos resultados dos sete itens, em que o mínimo é " 0 " e o máximo " 21 ". As notas mais elevadas em cada escala correspondem a estados afetivos mais negativos.

$\mathrm{O}$ último instrumento utilizado foi o questionário SF-36, validado para a cultura brasileira por Ciconelli ${ }^{20}$. Trata-se de 11 perguntas fechadas, com 36 itens e tendo como propósito avaliar 8 domínios divididos em 2 grupos: o físico, envolvendo a capacidade funcional, aspectos físicos, dor e estado geral da saúde e o Mental envolvendo saúde mental, aspectos emocionais, sociais e vitalidade. Cada domínio recebe um escore que varia de 0 a 100, que corresponde do pior ao melhor estado de saúde ${ }^{21}$.

\section{PROCED I MENTO}

\section{Coleta de dados}

Os instrumentos de medidas foram aplicados durante o mês de março de
2016, em três ambientes diferentes, sendo eles um ambiente próximo a uma Universidade, um ambiente de lazer e uma praça pública do município.

As entrevistas dos questionários ocorreram sem controle de tempo para o correto e completo preenchimento, a fim de que não houvesse razões de conduzir o voluntário a respostas apressadas. O tempo médio gasto com a aplicação dos questionários foi de 15 minutos.

\section{Análise dos dados}

Inicialmente, foi realizada uma análise descritiva para os dados, utilizando-se distribuições de frequências para os dados qualitativos. Nas análises dos questionários, EADS-21 e SF-36, primeiramente foram construídos os escores dos mesmos e aplicou-se o teste de Shapiro-Wilk para verificar se os dados eram normalmente distribuídos. Em seguida, devido à não normalidade dos dados, utilizou-se o teste de Kruskal-Wallis para comparação dos grupos. Já para verificar a associação entre as variáveis qualitativas fez-se uso do teste de Qui-quadrado e, com intuito de quantificar a relação entre as categorias, foram calculadas as razões de prevalências (RP) com os respectivos intervalos de confiança de 95\%. Em todos os testes utilizou-se um nível de significância de 5\%.

\section{CONSIDERAÇÕES ÉTICAS}

O presente estudo foi submetido e conduzido dentro dos padrões exigidos pelo Comitê de Ética em Pesquisa em Seres Humanos da Faculdade de Fisioterapia-UNIC de Cuiabá-MT, sendo que todos os sujeitos que participaram do estudo leram e assinaram o Termo de Consentimento Livre e Esclarecido.

\section{RESULTADOS}

Considerando o grau de severidade da disfunção temporomandibular (DTM), verificou-se que $48,3 \%$ dos indivíduos entrevistados apresentavam DTM leve, enquanto que aproximadamente $37 \%$ dos indivíduos foram classificados como livres de DTM, 9,2\% com DTM moderada e 5,8\% com DTM severa, totalizando uma prevalência da DTM de $63,3 \%$ (Tabela 1 ). Ainda, verificou-se que $75 \%$ dos indivíduos do sexo feminino apresentavam algum 
grau de DTM, enquanto esse percentual cai para 51,7\% quando considerado o indivíduo do sexo masculino (valor-p = 0,0095). Quando considerado se o indivíduo apresentava ou não companheiro, sua ocupação e a religião há uma predominância de DTM para indivíduos com companheiro $(64,7 \%)$, que estudam e trabalham/estudam (70\%) e não católicos (68,6\%). De acordo com o questionário para caracterização sociodemográfica, a média de idade dos indivíduos foi de 27 $(7,1)$ anos.

Nas Tabelas 2 e 3 são apresentadas as estatísticas descritivas (média, mediana e desvio padrão) em relação aos questionários EADS-21 e SF-36, respectivamente.

A partir da Tabela 2, observa-se que os Tabela 1 - Informações sociodemográficas por grupo de DTM

\begin{tabular}{|c|c|c|c|c|c|c|c|c|c|c|}
\hline \multirow{2}{*}{ Sexo } & \multicolumn{2}{|c|}{ DTM severa } & \multicolumn{2}{|c|}{ DTM moderada } & \multicolumn{2}{|c|}{ DTM leve } & \multicolumn{2}{|c|}{ Sem DTM } & \multicolumn{2}{|c|}{ Total } \\
\hline & & & & & & & & & & \\
\hline Feminino & 6 & $10,00 \%$ & 11 & $18,3 \%$ & 28 & $46,7 \%$ & 15 & $25,0 \%$ & 60 & $100 \%$ \\
\hline Masculino & 1 & $1,70 \%$ & 0 & $0,0 \%$ & 30 & $50,0 \%$ & 29 & $48,3 \%$ & 60 & $100 \%$ \\
\hline \multicolumn{11}{|l|}{ Companheiro } \\
\hline Com & 5 & $14,70 \%$ & 4 & $11,8 \%$ & 13 & $38,2 \%$ & 12 & $35,3 \%$ & 34 & $100 \%$ \\
\hline Sem & 2 & $2,30 \%$ & 7 & $8,1 \%$ & 45 & $52,3 \%$ & 32 & $37,2 \%$ & 86 & $100 \%$ \\
\hline \multicolumn{11}{|l|}{ Ocupação } \\
\hline Estudante & 3 & $7,50 \%$ & 3 & $7,5 \%$ & 22 & $55,0 \%$ & 12 & $30,0 \%$ & 40 & $100 \%$ \\
\hline Trabalha & 1 & $2,50 \%$ & 3 & $7,5 \%$ & 16 & $40,0 \%$ & 20 & $50,0 \%$ & 40 & $100 \%$ \\
\hline Trab./Est. & 3 & $7,50 \%$ & 5 & $12,5 \%$ & 20 & $50,0 \%$ & 12 & $30,0 \%$ & 40 & $100 \%$ \\
\hline \multicolumn{11}{|l|}{ Religião } \\
\hline Católica & 2 & $3,00 \%$ & 4 & $6,1 \%$ & 33 & $50,0 \%$ & 27 & $40,9 \%$ & 66 & $100 \%$ \\
\hline Não católica & 5 & $9,30 \%$ & 7 & $13,0 \%$ & 25 & $46,3 \%$ & 17 & $31,5 \%$ & 54 & $100 \%$ \\
\hline Total & 7 & $5,80 \%$ & 11 & $9,2 \%$ & 58 & $48,3 \%$ & 44 & $36,7 \%$ & 120 & $100 \%$ \\
\hline
\end{tabular}

Fonte: Elaborado pelos autores

Tabela 2 - Estatísticas descritivas do questionário EADS-21 por grupo de DTM

\begin{tabular}{lllll}
\hline \hline & DTM severa & DTM moderada & DTM leve & Sem DTM \\
Ansiedade & & & & \\
Média (dp) & $5,0(2,8)$ & $5,2(4,7)$ & $3,7(3,7)$ & $2,5(2,9)$ \\
Mediana & $6,0^{\mathrm{a}}$ & $5,0^{\mathrm{a}}$ & $2,5^{\mathrm{ab}}$ & $2,0^{\mathrm{b}}$ \\
$\begin{array}{l}\text { Depressão } \\
\text { Média (dp) }\end{array}$ & $7,0(4,8)$ & $4,6(4,0)$ & $3,5(3,8)$ & $2,3(3,7)$ \\
Mediana & $6,0^{\mathrm{a}}$ & $4,0^{\mathrm{ab}}$ & $2,5^{\mathrm{b}}$ & $1,0^{\mathrm{c}}$ \\
Stress & & & & \\
Média (dp) & $8,4(4,9)$ & $6,5(4,0)$ & $5,9(3,9)$ & $4,2(4,6)$ \\
Mediana & $9,0^{\mathrm{a}}$ & $6,0^{\mathrm{a}}$ & $5,0^{\mathrm{a}}$ & $3,0^{\mathrm{b}}$ \\
\hline \hline
\end{tabular}

Fonte: Elaborado pelos autores

indivíduos sem DTM apresentam valores menores nas três situações (ansiedade, depressão e stress) e maiores para DTM severa. Para verificar se essas diferenças são realmente significativas, utilizou-se o teste não paramétrico de Kruskal-Wallis e os valores-p determinados foram: 0,0355, 0,0022 e 0,0091 para ansiedade, depressão e stress, respectivamente. Como todos os casos foram significativos (valor-p < 0,05), realizou-se o teste de comparação múltipla para cada uma das situações. Em relação à ansiedade, obteve-se diferença significativa entre o grupo Sem DTM e os grupos com DTM moderada ou severa. A situação de stress comportou-se da mesma maneira que a ansiedade e a situação de depressão, constatou-se diferença significativa entre o grupo Sem DTM e todos os 
OLIVEIRA JÚNIOR GJ CRUZ JN DITOS L

CANDIDO LNS

CALDAS LF

ASSOCIAÇÃO ENTRE

OS SINTOMAS

DA DISFUNÇÃO

TEMPOROMANDIBULAR

E SUA RELAÇÃO

COM FATORES

PSICOLÓGICOS EM

COMUNIDADES DE

CUIABÁ-MT

36

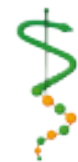

REV, ODONTOL.

UNIV, CID, SÃO

PAULO

$2016 ; 29(1): 32$ -

41, JAN-ABR grupos com DTM e também entre o grupo

DTM leve e o grupo DTM severa.

$\mathrm{Na}$ Tabela 3 a situação inverte-se em relação à Tabela 2, pois, quanto maior o resultado obtido pelo indivíduo, melhor é sua situação. Tem-se novamente que o grupo Sem DTM apresenta as maiores notas em todos os 8 casos estudados, e o que chama a atenção é que nem sempre os indivíduos do grupo DTM severa atingem as notas mais baixas. Os resultados referentes aos testes de Kruskal-Wallis para comparação dos 4 grupos (Sem DTM, DTM leve, DTM moderada e DTM severa), obtiveram o valor-p $<0,05$ para todos os casos considerados, mostrando que existe pelo menos uma diferença entre os grupos, por isso, novamente foi realizado o teste de comparações múltiplas para verificar quais pares de grupos diferiam entre si.

Para o caso da Capacidade funcional e Dor a diferença significativa ocorreu entre o grupo Sem DTM e todos os grupos com DTM; em relação ao Limitação dos aspectos físico e emocional a diferença significativa foi em relação ao grupo Sem DTM e ao grupo DTM moderada; quanto à Vitalidade e Estado geral de saúde ocorreu diferença significativa entre os grupos Sem DTM ou DTM leve e o grupo DTM moderado; a Saúde mental e o Aspecto social apresentaram diferença significativa entre o grupo Sem DTM e os grupos DTM moderada ou severa.

Com o objetivo de averiguar a associação entre as variáveis do questionário EADS-21 e do SF-36 com a variável presença (ausência) de DTM, realizou-se o teste de Qui-quadrado. Para a realização do teste, as variáveis quantitativas de ambos os questionários foram categorizadas em: "baixa" para os indivíduos que apresentaram escores abaixo da média e "alta" para os indivíduos que apresentaram escores maior ou igual à média. Uma vez que o número de indivíduos com DTM moderada e severa foi baixo, fez-se necessário uma recategorização, considerando agora 2 grupos - S,em DTM e com DTM (incluindo os indivíduos com DTM leve, DTM moderada e DTM severa).

Na Tabela 4 são apresentados os resultados dos testes considerando as variáveis do questionário EADS-21 (Ansiedade, De- pressão e Stress), bem como as razões de prevalência (RP).

Ressalta-se que, quanto menor o escore obtido no questionário, menos afetado pela doença o indivíduo se encontra e, portanto, a categoria "baixa" se encontra como base para RP. Observa-se que somente a variável ansiedade não está associada à variável presença (ausência) de DTM (valor-p $=0,0597$ ) e destaca-se que indivíduos classificados com depressão alta tiveram uma prevalência de DTM 45\% maior que aqueles com depressão baixa e aqueles com stress alto possuem prevalência de DTM 35\% maior que aqueles classificados com stress baixo (Tabela 4).

Para o questionário SF-36, observou-se que as variáveis Capacidade Funcional, Dor, Estado Geral de Saúde, Vitalidade, Aspecto Social, Limitação do Aspecto Emocional e Saúde Mental estão associadas à presença de DTM, conforme pode ser visto na Tabela 5. Nesse caso, verifica-se que, quanto maior for o escore obtido pelo indivíduo, melhor o mesmo se encontra em cada categoria e, portanto, a categoria alta se encontra como base para a RP. Destaca-se que indivíduos com Aspecto Social baixo tiveram uma prevalência de DTM 76\% maior que aqueles com Aspecto Social alto. Razões de prevalência semelhantes foram encontradas para a Capacidade funcional, na qual a prevalência de DTM entre aqueles com baixa capacidade é 1,75 vezes a prevalência daqueles com alta capacidade e também para o Estado geral de saúde $(1,73)$. Para as demais variáveis, as razões de prevalência foram menores.

\section{I S CUSSÃO}

No presente estudo constatou-se que não existe diferença quanto à idade entre os grupos com DTM () e Sem DTM (), concordando com alguns estudos já realizados $^{22-24}$. Ainda, a faixa de maior expressão de portadores de DTM foi de 21-30 anos $(64 \%)$, que é tida como a mais comum $^{25}$. Além disso, evidenciou-se nesta pesquisa a elevada prevalência de DTM em mulheres (75\%), sendo que $10 \%$ eram portadoras de DTM severa. Essa elevada prevalência, de acordo com Vedolin et al. 
Tabela 3 - Estatísticas descritivas do questionário SF-36 por grupo de DTM

\begin{tabular}{|c|c|c|c|c|}
\hline & DTM severa & DTM moderada & DTM leve & Sem DTM \\
\hline \multicolumn{5}{|c|}{ Capacidade funcional } \\
\hline Média (dp) & $67,1(18,5)$ & $70,5(24,6)$ & $82,7(17,6)$ & $93,3(10,2)$ \\
\hline Mediana & $65,0^{c}$ & $70,0^{\mathrm{bc}}$ & $85,0^{\mathrm{b}}$ & $95,0^{\mathrm{a}}$ \\
\hline \multicolumn{5}{|c|}{ Limitador de Aspecto Físico } \\
\hline Média (dp) & $53,6(33,6)$ & $34,1(39,2)$ & $66,4(36,1)$ & $78,4(25,1)$ \\
\hline Mediana & $50,0^{\mathrm{ab}}$ & $25,0^{\mathrm{b}}$ & $75,0^{\mathrm{a}}$ & $75,0^{\mathrm{a}}$ \\
\hline \multicolumn{5}{|l|}{ Dor } \\
\hline Média (dp) & $50,0(12,9)$ & $48,2(23,2)$ & $62,4(18,9)$ & $75,9(18,7)$ \\
\hline Mediana & $50,0^{\text {bc }}$ & $40,0^{c}$ & $60,0^{\mathrm{b}}$ & $70,0^{\mathrm{a}}$ \\
\hline \multicolumn{5}{|c|}{ Estado Geral de Saúde } \\
\hline Média (dp) & $66,4(19,7)$ & $43,6(23,4)$ & $64,7(18,6)$ & $78,6(14,8)$ \\
\hline Mediana & $75,0^{\mathrm{ab}}$ & $55,0^{c}$ & $67,5^{\mathrm{b}}$ & $80,0^{\mathrm{a}}$ \\
\hline \multicolumn{5}{|l|}{ Vitalidade } \\
\hline Média (dp) & $55,7(19,7)$ & $53,2(10,1)$ & $60,3(19,6)$ & $71,9(14,9)$ \\
\hline Mediana & $60,0^{\mathrm{b}}$ & $50,0^{\mathrm{b}}$ & $60,0^{\mathrm{b}}$ & $75,0^{\mathrm{a}}$ \\
\hline \multicolumn{5}{|c|}{ Aspecto social } \\
\hline Média (dp) & $46,4(32,0)$ & $51,1(25,3)$ & $72,2(24,2)$ & $83,0(17,7)$ \\
\hline Mediana & $37,5^{c}$ & $50,0^{c}$ & $75,0^{\mathrm{b}}$ & $87,5^{\mathrm{a}}$ \\
\hline \multicolumn{5}{|c|}{ Limitador de Aspecto Emocional } \\
\hline Média (dp) & $38,1(44,8)$ & $30,3(37,9)$ & $64,4(38,4)$ & $84,1(26,4)$ \\
\hline Mediana & $33,3^{\mathrm{bc}}$ & $10,0^{c}$ & $66,7^{\mathrm{b}}$ & $100,0^{\mathrm{a}}$ \\
\hline \multicolumn{5}{|c|}{ Saúde mental } \\
\hline Média (dp) & $53,1(19,1)$ & $52,7(24,3)$ & $68,9(19,6)$ & $79,1(15,8)$ \\
\hline Mediana & $44,0^{c}$ & $60,0^{c}$ & $72,0^{b}$ & $84,0^{\mathrm{a}}$ \\
\hline
\end{tabular}

Tabela 4 - Associação entre a presença de DTM e as variáveis do questionário EADS-21

\begin{tabular}{|c|c|c|c|c|c|c|c|c|c|}
\hline & \multicolumn{2}{|c|}{ COM DTM } & \multicolumn{2}{|c|}{ SEM DTM } & \multicolumn{2}{|c|}{ Total } & \multirow{2}{*}{ RP } & \multirow{2}{*}{ IC 95\% } & \multirow{2}{*}{ Valor P } \\
\hline & $\mathrm{n}$ & $\%$ & $\mathrm{n}$ & $\%$ & $\mathrm{n}$ & $\%$ & & & \\
\hline \multicolumn{10}{|l|}{ Ansiedade } \\
\hline Média (dp) & & & 3,44 & $(3,57)$ & & & & & \\
\hline Baixa & 44 & 57,14 & 33 & 42,86 & 77 & 100,00 & 1,00 & \multirow{2}{*}{$(1,00 ; 1,69)$} & \multirow{2}{*}{0,0597} \\
\hline Alta & 32 & 74,42 & 11 & 25,58 & 43 & 100,00 & 1,30 & & \\
\hline \multicolumn{10}{|l|}{ Depressão } \\
\hline Média (dp) & & & 3,39 & $(3,94)$ & & & & & \\
\hline Baixa & 42 & 54,55 & 35 & 45,45 & 77 & 100,00 & 1,00 & \multirow{2}{*}{$(1,12 ; 1,87)$} & \multirow{2}{*}{$0,0075^{*}$} \\
\hline Alta & 34 & 79,07 & 9 & 20,93 & 43 & 100,00 & 1,45 & & \\
\hline \multicolumn{10}{|l|}{ Stress } \\
\hline Média (dp) & & & \multicolumn{2}{|c|}{$5,51(4,32)$} & & & & & \\
\hline Baixa & 38 & 55,07 & 31 & 44,93 & 69 & 100,00 & 1,00 & \multirow{2}{*}{$(1,04 ; 1,77)$} & \multirow{2}{*}{$0,0289 *$} \\
\hline Alta & 38 & 74,51 & 13 & 25,49 & 51 & 100,00 & 1,35 & & \\
\hline
\end{tabular}

OLIVEIRA JÚNIOR GJ CRUZ JN

DITOS L

CANDIDO LNS

CALDAS LF

ASSOCIAÇÃO ENTRE

OS SINTOMAS

DA DISFUNÇÃO

TEMPOROMANDIBULAR

e sUa RELAÇÃo

COM FATORES

PSICOLÓGICOS EM

COMUNIDADES DE

CUIABÁ-MT 
I SSN $1983-5183$

OLIVEIRA JÚNIOR GJ CRUZ JN DITOS L

CANDIDO LNS

CALDAS LF

ASSOCIAÇÃO ENTRE OS SINTOMAS DA DISFUNÇÃO TEMPOROMANDI BULAR E SUA RELAÇÃO COM FATORES PSICOLÓGICOS EM COMUNIDADES DE CUIABÁ-MT

Tabela 5 - Associação entre a presença de DTM e as variáveis do questionário SF-36

\begin{tabular}{cccccccc}
\hline \hline COM DTM & \multicolumn{2}{c}{ SEM DTM } & Total & & RP & IC 95\% & Valor P \\
\hline & $\mathrm{n}$ & $\%$ & $\mathrm{n}$ & $\%$ & $\mathrm{n}$ & $\%$ & \\
\hline
\end{tabular}

Capacidade Funcional

Média

(dp)

$84,54(17,89)$

$\begin{array}{llllllllll}\text { Baixa } & 34 & 89,47 & 4 & 10,53 & \mathbf{3 8} & \mathbf{1 0 0 , 0 0} & 1,75 & (1,37 ; 2,22) & 0,0001^{*} \\ \text { Alta } & 42 & 51,22 & 40 & 48,78 & \mathbf{8 2} & \mathbf{1 0 0 , 0 0} & 1,00 & (1,37\end{array}$

Limitação do Aspecto Físico

Média

(dp)

$\begin{array}{llllllllll}\text { Baixa } & 34 & 73,91 & 12 & 26,09 & \mathbf{4 6} & \mathbf{1 0 0 , 0 0} & 1,30 & \\ \text { Alta } & 42 & 56,76 & 32 & 43,24 & \mathbf{7 4} & \mathbf{1 0 0 , 0 0} & 1,00 & (1,00 ; 1,69) & 0,0579 \\ \text { Dor } & & & & & & & & & \end{array}$

Média

(dp)

$65,33(20,90)$

$\begin{array}{llllllll}\text { Baixa } & 46 & 79,31 & 12 & 20,69 & 58 & \mathbf{1 0 0 , 0 0} & 1,65\end{array}$

$\begin{array}{llllllll}\text { Alta } & 30 & 48,39 & 32 & 51,61 & \mathbf{6 2} & \mathbf{1 0 0 , 0 0} & 1,00\end{array}$

$(1,23 ; 2,19) \quad 0,0004^{*}$

Estado Geral de Saúde

Média

(dp)

$67,95(20,31)$

$\begin{array}{llllllll}\text { Baixa } & 42 & 84,00 & 8 & 16,00 & 50 & \mathbf{1 0 0 , 0 0} & 1,71\end{array}$

$\begin{array}{llllllll}\text { Alta } & 34 & 48,57 & 36 & 51,43 & 70 & \mathbf{1 0 0 , 0 0} & 1,00\end{array}$

$(1,32 ; 2,26) \quad 0,0001^{*}$

Vitalidade

Média

(dp)

$63,67(18,35)$

$\begin{array}{llllllll}\text { Baixa } & 43 & 81,13 & 10 & 18,87 & 53 & \mathbf{1 0 0 , 0 0} & 1,65\end{array}$

Alta 33

Aspecto Social

Média

(dp)

$72,70(24,95)$

$\begin{array}{llllllll}\text { Baixa } & 43 & 84,31 & 8 & 15,69 & 51 & \mathbf{1 0 0 , 0 0} & 1,75\end{array}$

$\begin{array}{llllllll}\text { Alta } & 33 & 47,83 & 36 & 52,17 & \mathbf{6 9} & \mathbf{1 0 0 , 0 0} & 1,00\end{array}$

$(1,34 ; 2,32)<0,0001^{*}$

Limitação do Aspecto Emocional

Média

(dp)

$66,94(38,29)$

$\begin{array}{llllllll}\text { Baixa } & 47 & 77,05 & 14 & 22,95 & \mathbf{6 1} & \mathbf{1 0 0 , 0 0} & 1,57\end{array}$

$\begin{array}{llllllll}\text { Alta } & 29 & 49,15 & 30 & 50,85 & \mathbf{5 9} & \mathbf{1 0 0 , 0 0} & 1,00\end{array}$

$(1,17 ; 2,10) \quad 0,0015^{*}$

Saúde Mental

Média

(dp)

$70,23(20,41)$

$\begin{array}{llllllll}\text { Baixa } & 41 & 78,85 & 11 & 21,15 & 52 & \mathbf{1 0 0 , 0 0} & 1,55\end{array}$

$\begin{array}{llllllll}\text { Alta } & 35 & 51,47 & 33 & 48,53 & \mathbf{6 8} & \mathbf{1 0 0 , 0 0} & 1,00\end{array}$

$(1,17 ; 2,01) \quad 0,0020^{*}$
UNIV, CID, SÃO PAULO $2016 ; 29(1): 32-$ 41, JAN-ABR 
${ }^{26}$, deve estar relacionada às diferenças fisiológicas do gênero, tais como: variações hormonais, estrutura muscular e limiar e de dor mais baixo.

Neste trabalho utilizaram-se as categorias com companheiro e sem companheiro, sendo que aquela apresentou um número maior, embora com diferença pequena, de indivíduos com DTM, opondo-se ao trabalho de Kliemann, Brunetti e Oliveira ${ }^{22}$, que observaram uma prevalência maior de indivíduos com estados civis solteiro e divorciado quando comparados aos casados.

Um outro fator, listado como desencadeante nos distúrbios da DTM $^{27}$, aqui considerado, é o fator ocupacional. Entre os selecionados em quantidades iguais para cada categoria, as categorias trabalha/estuda e estuda apresentaram um percentual alto $(70 \%)$ de indivíduos com DTM. A diferença entre tais categorias está no percentual que apresentam DTM moderada ou severa, sendo $20 \%$ para categoria estuda/trabalha e $15 \%$ para categoria estuda, evidenciando que o stress e a ansiedade produzidos pelo desgaste do trabalho e estudo são variáveis de suma importância na identificação desse distúrbio.

$\mathrm{Na}$ literatura, os fatores emocionais como estresse, depressão e ansiedade são referidos como fatores causadores da DTM $^{28-31}$. Para o presente estudo, as variáveis estresse e depressão conservam esta afirmação. Embora a variável Ansiedade não tenha apresentado associação com a DTM, esta não pode ser desconsiderada, uma vez que apresentou um valor-p muito próximo do nível de significância considerado. Assim, para um resultado mais conclusivo com respeito a essa associação, mais estudos deverão ser conduzidos.

Para o questionário SF-36, considerando seus dois grandes grupos, físico e mental (Capacidade Funcional, Limitador do Aspecto Físico, Dor, Estado Geral de
Sáude, Vitalidade, Aspecto Social, Limitador do Aspecto Físico, Saúde mental), verificaram-se médias de escores menores em indivíduos com DTM (59 (físico) e 54 (mental) para os indivíduos com DTM e médias de 82 (físico) e 80 (mental) para os indivíduos sem DTM). Esse fato vai ao encontro do estudo de Venâncio e Camparis $^{29}$, no qual foi verificada uma média de qualidade de vida para o grupo sem DTM maior que aquelas observadas para o grupo com DTM. Ainda, de acordo com Roberto et al. ${ }^{32}$, existe uma relação inversamente proporcional entre Qualidade de vida e DTM, podendo-se dizer que, quanto menor ou ausente a DTM, maior a qualidade de vida.

\section{CONCLUSÃO}

Os resultados deste estudo revelaram uma elevada prevalência de DTM para indivíduos do sexo feminino. Ainda, há maior predominância de DTM em indivíduos com companheiro que trabalham e estudam e não católicos. Ao se relacionar essa variável com as variáveis do EADS21, verificou-se uma associação significativa desta com depressão e stress. Quando consideradas as variáveis do questionário SF-36, a presença de DTM esteve associada às variáveis capacidade funcional, dor, estado geral de saúde, vitalidade, aspecto social, Limitação do aspecto emocional e saúde mental.

Este trabalho é um estudo preliminar, realizado considerando um tamanho amostral reduzido. Contudo, os resultados indicaram que a prevalência dessa doença pode ser alta. Assim sendo, a divulgação desse assunto junto as populações sujeitas a grande stress e a grande esforço mental, como são os indivíduos que trabalham e estudam, torna-se de grande relevância para que os mesmos atentem os sinais e sintomas de DTM e busquem ajuda para solucioná-los. oliveira JÚNIOR GJ CRUZ JN

DITOS L

CANDIDO LNS

CALDAS LF

ASSOCIAÇÃO ENTRE

OS SINTOMAS

DA DISFUNÇÃO

TEMPOROMANDI BULAR

E SUA RELAÇÃO

COM FATORES

PSICOLÓGICOS EM

COMUNIDADES DE

CUIABÁ-MT
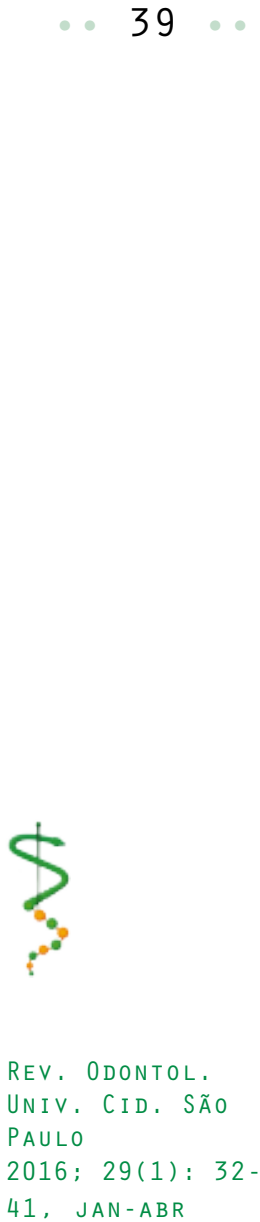
OLIVEIRA JÚNIOR GJ CRUZ JN DITOS L

CANDIDO LNS

CALDAS LF

ASSOCIAÇÃO ENTRE OS SINTOMAS

DA DISFUNÇÃO TEMPOROMANDIBULAR

E SUA RELAÇÃO

COM FATORES

PSICOLÓGICOS EM

COMUNIDADES DE

CUIABÁ-MT

40

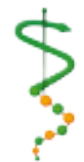

REV. ODONTOL.

UNIV, CID, SÃO

PAULO

$2016 ; 29(1): 32$ -

41, JAN-ABR

\section{REFERÊNCIAS}

1. Savalle W. Anatomia do aparelho mastigatório. In: Steenks M, Wijer A, editors. Disfunções da articulação temporomandibular do ponto de vista da Fisioterapia e da Odontologia: diagnóstico e tratamento. São Paulo: Santos; 1996.

2. Okeson J. Fundamentos de oclusão e desordens temporomandibulares. São Paulo: Artes Médicas; 1992.

3. Smith L, Weiss E, Lehmkuhl D. Cinesiologia clínica de Brunnstrom. 5 ed. São Paulo: Manole; 1997.

4. Mascarenhas M, Dutra L. Inter-relações funcionais entre ATM e coluna cervical. In: Maciel R, editor. ATM e dores craniofaciais: fisiopatologia básica. São Paulo: Santos; 2003.

5. Carrara SV, Conti PCR, Barbosa JS. Termo do $1^{\circ}$ Consenso em Disfunção Temporomandibular e Dor Orofacial. Dental Press Journal of Orthodontics. 2010;15(3):114-20.

6. Donnarumma MDC, Muzilli CA, Ferreira C, Nemr K. Disfunções temporomandibulares: sinais, sintomas e abordagem multidisciplinar. Revista CEFAC. 2010;12(5):788-94.

7. Landulpho AB, Silva WAB, Silva FA. Análise dos ruídos articulares em pacientes com disfunção temporomandibular tratados com aparelhos interoclusais. JBA j bras oclusão ATM dor orofac. 2003;3(10):112-7.

8. Silva FA, Lameira AG, Bérzin F, Silva WAB. Tratamento das alterações funcionais do sistema estomatognático. Rev Assoc Paul Cir Dent. 1993;47(3):1055-62.

9. Grade R, Caramês J, Pragosa A, Carvalhão J, Sousa S. Postura e disfunção temporo-mandibular: controvérsias actuais. Revista Portuguesa de Estomatologia, Medicina Dentária e Cirurgia Maxilofacial. 2008;49(2):111-7.

10. Okeson JP. Management of temporomandibular disorders and occlusion: Elsevier Health Sciences; 2014.
11. Manfredini D, Bandettini di Poggio A, Cantini E, Dell'Osso L, Bosco M. Mood and anxiety psychopathology and temporomandibular disorder: a spectrum approach. Journal of oral rehabilitation. 2004;31(10):933-40.

12. Brasio KM, Laloni DT, Fernandes QP, Bezerra TdL. Comparação entre três técnicas de intervenção psicológica para tratamento da fibromialgia: treino de controle de stress, relaxamento progressivo e reestruturação cognitiva. Rev Ciências Medicas. 2003;12(10):307-18.

13. Lipp M. O modelo quadrifásico do estresse. In: Lipp M, editor. Mecanismos neuropsicofisiológicos do estresse: teoria e aplicações clínicas. São Paulo Casa do Psicólogo; 2003. p. 17-22.

14. Lipp M, Malagris L. O stress emocional e seu tratamento. In: Rangé $B$, editor. Psicoterapias cognitivo-comportamentais: um diálogo com a psiquiatria. Porto Alegre:: Artmed; 2001.

15. Domingos N, Lipp M. Stress em pacientes candidatos a transplante de fígado. In: Miyazaki M, Domingos N, Nelson I, editors. Psicologia da saúde: pesquisa e prática. São José do Rio Preto: THS/Arantes 2006.

16. Malagris LEN, Fiorito ACC. Avaliação do nível de stress de técnicos da área de saúde. Estudos de Psicologia (Campinas). 2006;23(4):391-8.

17. Dancey C, Reidy J. Estatística sem Matemática para Psicologia: usando SPSS para Windows. 3 ed. Porto Alegre: Artemd; 2006.

18. Nomura K, Vitti M, Oliveira ASd, Chaves TC, Semprini $M$, Siéssere $S$, et al. Use of the Fonseca's questionnaire to assess the prevalence and severity of temporomandibular disorders in brazilian dental undergraduates. Brazilian Dental Journal. 2007;18(2):163-7. 
19. Chaves TC, Oliveira ASd, Grossi DB. Principais instrumentos para avaliação da disfunção temporomandibular, parte I: índices e questionários; uma contribuição para a prática clínica e de pesquisa. Fisioterapia e Pesquisa. 2008;15(1):92-100.

20. Ciconelli R, Ferra M, Santos W, Meinão I, Quaresma M. Tradução para a língua portuguesa e validação do questionário genérico de qualidade de vida SF-36 (Brasil SF-36). Rev Bras Reumatol. 1999;39(3):143-50.

21. Martinez MC, Paraguay AIBB, Latorre MRDO. Relação entre satisfação com aspectos psicossociais e saúde dos trabalhadores. Revista de Saúde Pública. 2004;38(1):55-61.

22. Kliemann C, Brunetti R. Pacientes queixosos de disfunção crânio mandibular: avaliação de características pessoais e estresse em relação a não queixosos. RGO. 1998;46(1):7-10.

23. Lima D, Brunetti R, Oliveira W. Estudo da prevalência de disfunção craniomandibular segundo o índice de Helkimo tendo como variáveis: sexo, faixa etária e indivíduos tratados ou não ortodonticamente. RPG Revista de Pós-Graduação. 1999;2(2):28-35.

24. Teixeira VCB, Matias KS, Procópio ASF, Luz JGC. Sintomatologia das disfunções da articulação temporomandibular conforme o gênero e a faixa etária por meio dos índices de Helkimo. Rev odonto/ UNICID. 2003;15(3):1939.

25. Donegá SHdP, Cardoso R, Procópio ASF, Luz JGdC. Análise da sintomatologia em pacientes com disfunções intra-articulares da articulação temporomandibular. Rev odontol Univ São Paulo. 1997;11(supl):77-83.
26. Vedolin GM, Lobato VV, Conti PC, Lauris JR. The impact of stress and anxiety on the pressure pain threshold of myofascial pain patients. Journal of oral rehabilitation. 2009;36(5):31321.

27. Morais J, Bottino M. Papel da oclusão nos distúrbios da articulação temporomandibular. Revista da Faculdade de Odontologia de São José dos Campos. 1972(1):21-31.

28. Moreira MMSM, Alencar Junior FGPd, Bussadori CMC. Fatores psicológicos na etiologia da disfunção craniomandibular. Rev Assoc Paul Cir Dent. 1998;52(5):377-81.

29. Venancio RdA, Camparis CM. Estudo da relação entre fatores psicossociais e desordens têmporo-mandibulares. Rev bras odontol. 2002;59(3):152-4.

30. Rantala MA, Ahlberg J, Suvinen TI, Nissinen $M$, Lindholm $H$, Savolainen $\mathrm{A}$, et al. Temporomandibular joint related painless symptoms, orofacial pain, neck pain, headache, and psychosocial factors among non-patients. Acta odontologica Scandinavica. 2003;61(4):217-22.

31. Selaimen C, Brilhante DP, Grossi ML, Grossi PK. Depression and neuropsychological testing in patients with temporomandibular disorders. Ciência \& Saúde Coletiva. 2007;12(6):162939.

32. Deli R, Macrì LA, Mannocci A, La Torre G. Measuring Quality of Life in TMD: use of SF-36. Italian Journal of Public Health. 2012;6(2).

Recebido em 01/11/2016

Aceito em 01/12/2016
OLIVEIRA JÚNIOR GJ CRUZ JN

DItos L

CANDIDO LNS

CALDAS LF

ASSOCIAÇÃO ENTRE

OS SINTOMAS

DA DISFUNÇÃO

TEMPOROMANDIBULAR

e sua relação

COM FATORES

PSICOLÓGICOS EM

COMUNIDADES DE

CUIABÁ-MT

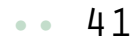

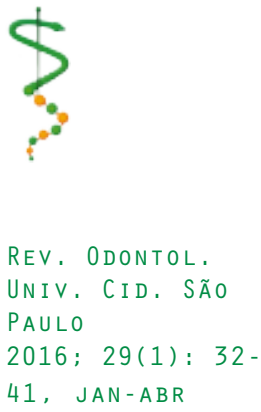

